\title{
In silico virtual screening-based study of nutraceuticals predicts the therapeutic potentials of folic acid and its derivatives against COVID-19
}

\author{
Vipul Kumar ${ }^{1} \cdot$ Sudhakar Kancharla $^{2} \cdot$ Manoj Kumar Jena $^{1} \mathbb{D}$
}

Received: 5 May 2020/Accepted: 11 November 2020/Published online: 28 January 2021

(C) Indian Virological Society 2021

\begin{abstract}
The recent outbreak of the novel coronavirus (SARS-CoV-2) in the Wuhan province of China has taken millions of lives worldwide. In this pandemic situation and absence of known drugs and vaccines against novel coronavirus disease (COVID-19), there is an urgent need for the repurposing of the existing drugs against it. So, here we have examined a safe and cheap alternative against this virus by screening hundreds of nutraceuticals compounds against known therapeutic targets of SARS-COV-2 by molecular docking. The virtual screening results were then analyzed for binding energy and interactive residues and compared with some already known hits in the best binding pose. All these analyses of this study strongly predicted the potential of Folic acid and its derivates like Tetrahydrofolic acid and 5-methyl tetrahydrofolic acid against SARSCOV-2. The strong and stable binding affinity of this water-soluble vitamin and its derivatives against the SARS-COV-2, indicating that they could be valuable drugs against the management of this COVID-19 pandemic. This
\end{abstract}

Electronic supplementary material The online version of this article (https://doi.org/10.1007/s13337-020-00643-6) contains supplementary material, which is available to authorized users.

Manoj Kumar Jena

manoj.20283@1pu.co.in

Vipul Kumar

vipu12732@gmail.com

Sudhakar Kancharla

sudhakar@devlabwerks.com

1 Department of Biotechnology, School of Bioengineering and Biosciences, Lovely Professional University, Phagwara, Punjab 144411, India

2 Devansh Lab Werks, 234 Aquarius Drive, Homewood, AL 35209, USA study could serve as the starting point for further investigation of these molecules through in vitro and in vivo assays.

Keywords SARS-CoV-2 - COVID-19 · Nutraceuticals · Molecular docking $\cdot$ Folic acid

\section{Introduction}

The outbreak of novel coronavirus disease (COVID-19) has now been declared as the pandemic worldwide by world health organization (WHO). COVID-19 outbreak happened in December 2019 in Wuhan province of China by a virus called Severe Acute Respiratory Syndrome related to Corona Virus-2 (SARS-COV-2) [9, 39].This disease has been spread throughout the world, which has infected more than 1 million people, and over 50 thousand people have been died (www.who.int). This virus has given a major challenge to the world medical facilities and economy.

Coronaviruses belong to the family of Coronaviridae, order Nidovirales and realm Riboviria [10]. This virus group contains large positive-sense RNA, around $30 \mathrm{~Kb}$ size, which is encapsulated in the membrane envelope [15]. The membrane of the coronavirus is occupied by the glycoprotein projections, which gives the crown-like appearance to the virus; hence the name is Coronavirus [42]. Coronaviruses are divided into four classes, namely alpha, beta, gamma and delta. The SARS-CoV2, Middle East Respiratory Syndrome Virus (MERS) and SARS-CoV are included in the beta class of the coronavirus [15, 29]. All the beta class viruses are known to attack the respiratory system of the human and cause pneumonia [35, 43]. In several cases, it is reported that SARS-CoV-2 can be 
responsible for multiple organ failure too [16, 28]. Dry cough, difficulty in breathing, fever, and tiredness are the major symptoms found in COVID-19 patients [27]. The current pandemic and number of reported cases are showing that SARS-COV-2 is more contagious and transmissive in comparison to MERS, SARS-CoV, and other previous coronaviruses [26, 49, 50].

SARS-CoV-2 encodes for various structural as well as non-structural proteins, which helps in the viral entry as well as replication inside the host cell. One of the major inducers of the host immune response is a structural protein that is embedded in the virus membrane known as Spike (S) glycoprotein $[1,52]$. The glycosylated S protein facilitates the entry of the virus into the host cell $[3,46]$. The $S$ protein binds to the host cell membrane receptor, Angiotensin-Converting Enzyme-2 (ACE-2), and further its invasion and fusion inside the host cell membrane is mediated by host Transmembrane Serine Protease (TMPRSS2) [4, 22]. Recently it also reported that the affinity of SARS-CoV-2 towards the ACE-2 is 10-20 times higher than SARS-CoV, which can be the reason for its higher severity in terms of transmission in comparison to previously known coronaviruses [14, 31]. Other than $\mathrm{S}$ glycoprotein, SARS-CoV-2 encodes various non-structural proteins, which include the main protease $\left(\mathrm{M}^{\mathrm{pro}} / 3 \mathrm{CL}^{\mathrm{pro}}\right)$, papain-like protease ( $\mathrm{PL}^{\mathrm{pro}}$ ), RNA dependent RNA polymerase (RdRp) and Nsp15 an endoribonuclease. These non-structural proteins help in the replication and life cycle of the virus inside the host cell. Once the Virus enters inside the cell, its ssRNA is translated into various polyproteins using host translation machinery, and these viral polyproteins are then cleaved into functional viral proteins by $\mathrm{M}^{\text {pro }}$ and $\mathrm{PL}^{\text {pro }}[8,33]$. These cleavage yields various Nsps, which exhibits multiple enzymatic activities. All the functions and roles of these cleaved Nsps are not clearly known, but some are specifically linked to the replication of the single stranded viral RNA. One such Nsps is Nsp15, which is RNA uridylate specific endoribonuclease associated with the viral RNA processing $[5,37] . \mathrm{RdRp}$ is an enzyme that makes a negative-strand RNA template for the synthesis of more viral RNAs [40].

To date, there are no such specific antiviral agents for the treatment of COVID-19 [13]. The fast and best option for its treatment is to repurpose already known drugs, natural molecules and nutraceuticals. Various nutraceutical compounds have reported to be beneficial against viruses $[19,20,48]$. Ascorbic acid or Vitamin C has been reported as beneficial against the influenza virus [21,24]. Similarly, resveratrol against herpes simplex virus (HSV) is well documented [2]. The effect of folic acid malnutrition on rotaviral infected mice has been shown through dose dependent manner [32]. Recently the role of folic acid in the management of COVID-19 by the prevention of SARS-
CoV 2 entry inside the human cells has been reported [51]. Nutraceuticals are safe to consume and have many antimicrobial activities reported to date [34], so taking these properties into consideration, we performed the molecular docking based virtual screening of the nutraceutical compounds against S protein-ACE2 complex, $\mathrm{M}^{\text {pro }}, \mathrm{PL}^{\text {pro }}$ and Nsp15, and compared with already known hit compounds. For Spike-ACE2 surface interaction inhibition, Arbidol (umifenovir), which is known to disrupt the ACE-2-Spike has been taken as positive control to compare the bioactivity of tested nutraceutical compounds. Arbidol is in clinical trial in China and it has been reported that its monotherapy resulted people to be COVI-19 negative within 14 days [47]. While, for PL ${ }^{\text {pro }}$, GRL0617 has been considered as positive control, as in recent study its IC50 against $\mathrm{PL}^{\text {pro }}$ was found to be $2.2 \pm 0.3 \mu \mathrm{mol} / \mathrm{L}$ [17]. Further in case of $\mathrm{M}^{\text {pro }}$, Pimozide is taken as positive control to compare the potential of nutraceuticals. The IC50 value of Pimozide against $\mathrm{M}^{\text {pro }}$ has been reported as $42 \pm 2 \mu \mathrm{mol} / \mathrm{L}$ [44]. Finally, for NSP15, Glisoxepide has been taken as positive control, its IC50 valued was reported as $9.2 \mu \mathrm{mol} / \mathrm{L}$ [7]. Analysis of the virtual screening results shows that folic acid (Vitamin B9) and its derivative were in the top three compounds, which had equivalent binding energy to positive control against all the proteins studied. Other than folic acid and its derivatives, Gingkoglide-A, a terpene lactone mainly found in tree Ginkgo biloba was found best against $\mathrm{PL}^{\text {pro }}$ and Magnesium ascorbate against $\mathrm{M}^{\text {pro }}$. Although screening of 106 nutraceuticals compounds against all these target proteins and getting folic acid and its derivatives in the top 3 lead compounds against every protein target indicates that these compounds could play a potential role in the prevention and management of the COVID-19.

\section{Materials and methods}

\section{Retrieval and preparation of protein structures}

The structure of all the SARS-CoV-2 targets was retrieved from the RCSB protein data bank. Four targets, namely $S$ protein (Receptor Binding Domain)-ACE2 complex, $\mathrm{M}^{\text {pro }}$, PL $^{\text {pro }}$, Nsp15 having PDB IDs 6LZG [45], 6LU7 [23], 6W9C (To be published), and 6W01 [25] respectively. The water molecules are removed, polar hydrogens were added, and non-essential heteroatoms were deleted as part of protein preparation before docking with the help of Discovery studio software 2020 [6]. 
Virtual screening of the nutraceuticals against SARS-CoV-2 proteins

A total of 106 compounds library of the latest release (2020-1) of nutraceutical was retrieved from the drug bank database (www.drugbank.ca) in SDF format. The compounds, namely, Arbidol (CID: 131411), GRL0617 (CID: 24941262), Pimozide (CID: 16362), and Glisoxepide (CID:32778), are retrieved from PubChem database and has been considered as positive control to compare the binding energy of nutraceuticals All these compounds were minimized using UFF forcefield with conjugate gradient protocol having 500 steps, with the help of PyRx software [12]. Then both proteins and ligands were converted to pdbqt file format for virtual screening using Autodock vina [41], inbuilt in PyRx software. The top 3 compounds have been reported against all the targets through this virtual screening study.

\section{Interactions of the ligand with critical residues}

Top 3 lead compounds based on the binding energy of the docked complex were analyzed for the visualization of the best binding pose. As the importance of hydrogen bonding is well documented in drug discovery and design, further hydrogen bond and other non-polar interactions of best binding poses were analyzed using Discovery studio software [6].

\section{Analysis and comparison of hit nutraceutical compounds with already known inhibitors}

The top lead nutraceutical compounds were than taken for the analysis of ADMET properties using SWISS ADME webserver [11]. The ADMET and drug likeness properties of top hit nutraceutical compounds were then compared with positive control to analysis the potential of hit molecules as drug candidate.

\section{Results}

\section{Folic acid and its derivatives can prevent the interaction of $S$ protein with ACE-2 receptor}

The 3D structure of the ACE2-Spike protein was retrieved from RCSB-PDB (PDB ID: 6LZG) which is resolved using $\mathrm{X}$-ray crystallography at $2.5 \AA$ resolution. In the crystal structure the C-terminal domain of SARS-CoV-2- spike (S) protein in complex with human ACE2 has been observed, which is revealing the ACE2-binding mode comparable to that observed for SARS-CoV. Prepared structure of the $\mathrm{S}$ protein (Receptor Binding Domain)-
ACE2 complex was first analyzed in the native conformation to investigate the major interactions between spike protein and ACE-2 receptor. In the analysis, it was found that the TYR41, ASP38, GLU35, ASP30 of ACE-2 receptor was making the hydrogen bond with THR500, GLY496, GLN493 and LYS417 of S protein respectively (Supplementary Fig. 1).Further, the complex was taken for the screening of nutraceuticals against and comparing the top hits binding energy and interactions with positive control Arbidol.. For the docking of the ligands, the grid was generated, taking interacting residues in the center of the box. The coordinates of the grid $X=-35.07$, $Y=25.72, Z=0.66$ and the dimension of the grid was 25 Angstrom $^{3}$. The ligands having the best binding affinity towards the complex were taken further for the analysis of their interacting residues. In the best docked pose the docking energy of Arbidol was $-6.2 \mathrm{kcal} / \mathrm{mol}$ and it was making hydrogen bond with ASP33 and HIS34 of ACE-2 (Fig. 1a). On the other hand, out of 106 nutraceuticals screened; Folic Acid had the strongest binding energy of $-9.0 \mathrm{kcal} / \mathrm{mol}$, followed by tetrahydrofolic acid $(-8.9 \mathrm{kcal} / \mathrm{mol})$ and 5-methyltetraydrofolic acid $(-8.6 \mathrm{kcal} / \mathrm{mol})$.The docking score of top hit nutraceutical compounds were showing better binding affinity than already known drug candidate Arbidol. The interaction analysis further showed that these nutraceuticals have the potential to break or prevent the interactions between the protein complex. Folic acid could break the hydrogen bond interaction Of ASP30 of ACE-2 with S protein; similarly, GLY496 of S protein, which previously interacting with ACE-2, was interacting with folic acid after docking. Likewise, GLY493, LYS417 of spike protein, was interacting with Folic acid (Fig. 1b). These significant reductions of the major interacting residues between the spike protein and ACE2 showing the potential of folic in the prevention of viral entry. Similarly, when we analyzed the Tertrahydrofolic acid interactions, we found that it was also able to break the crucial interactions and was making interactions with GLN493, GLN496 of spike protein and ASP38 of ACE-2 receptor (Supplementary Fig. 2A). Furthermore, 5-methyl-tetrahydrofolic acid was making the hydrogen bond with ASP30 of ACE-2 and LYS417 of S protein and was making various non-polar interactions with the crucial amino acids, which were previously helping the interaction of spike protein with ACE-2 receptor (Supplementary Fig. 2B). All these analyses of the binding energy and interactions with crucial residues of the complex indicating the possibility of folic acid and its derivatives to be lead molecules against SARS-CoV-2 entry inside the human cells.(Supplementary Table 1). Finally, the top hit compounds were investigated for ADMET properties and drug likeliness according to Lipinski rule of five and Ghose $[18,30]$. It was found that all the drug candidates were 
A
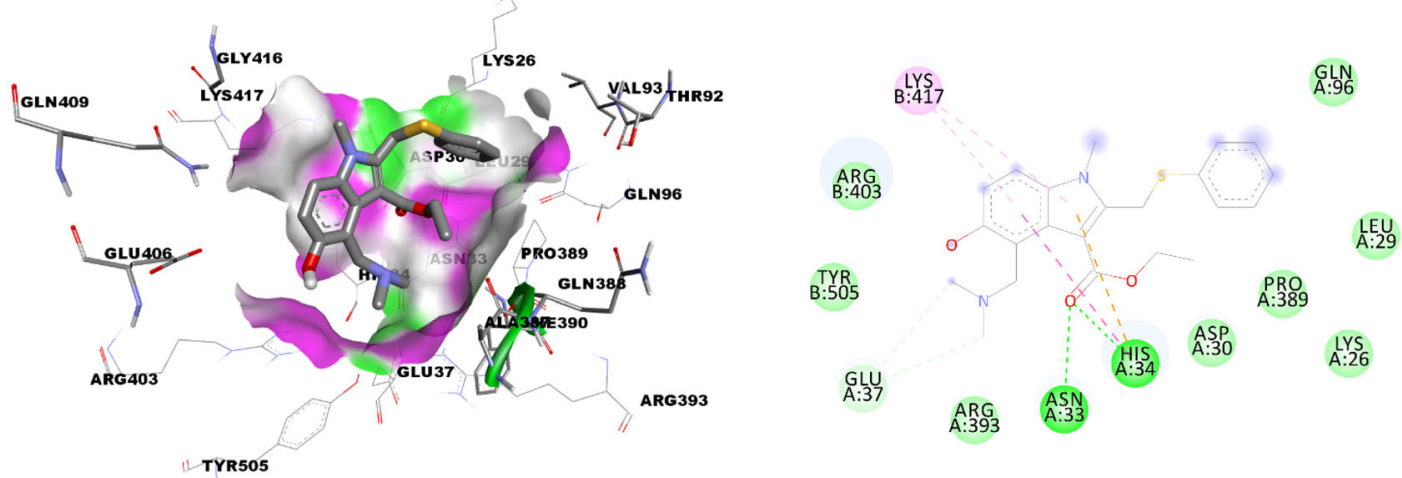

B
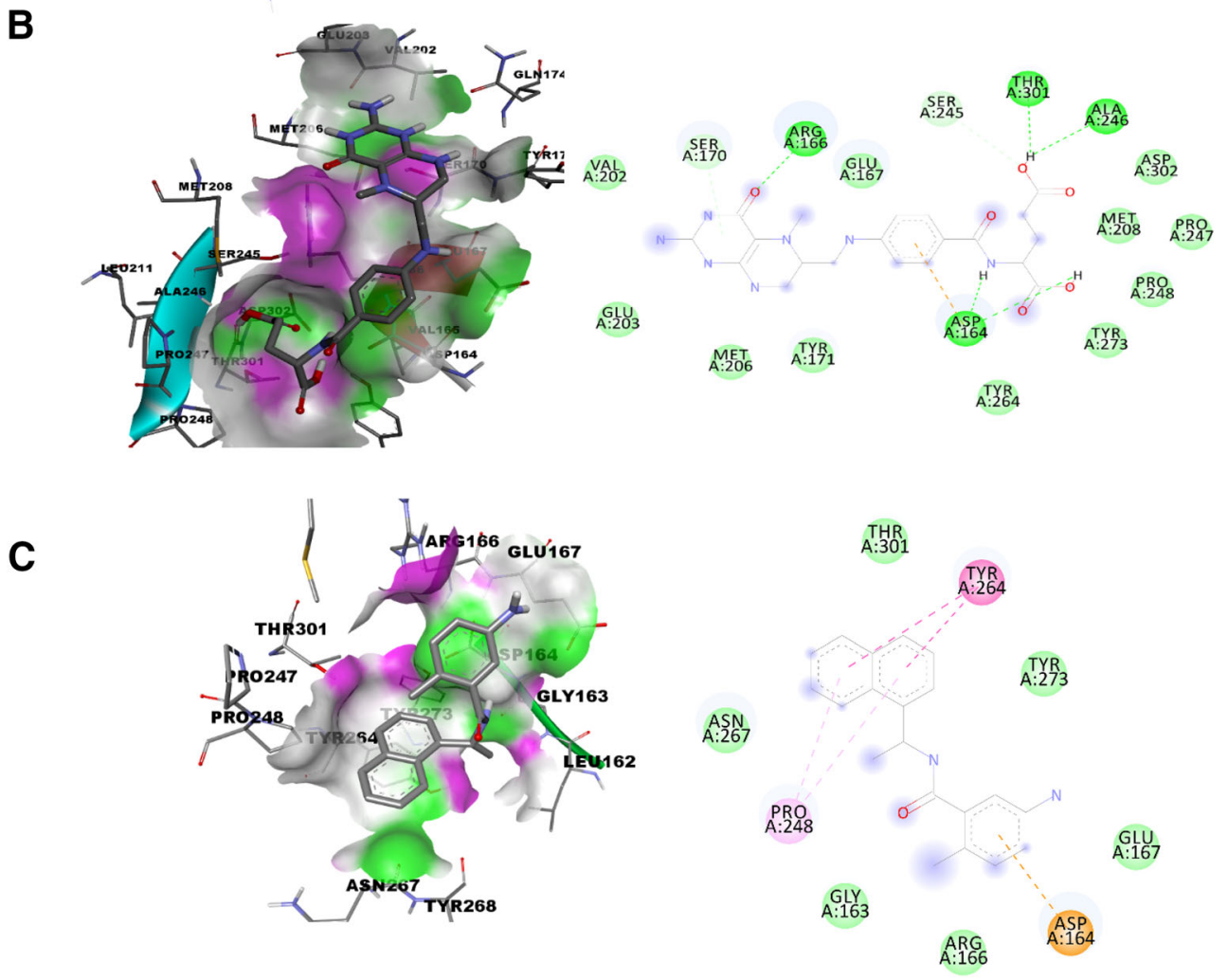

D

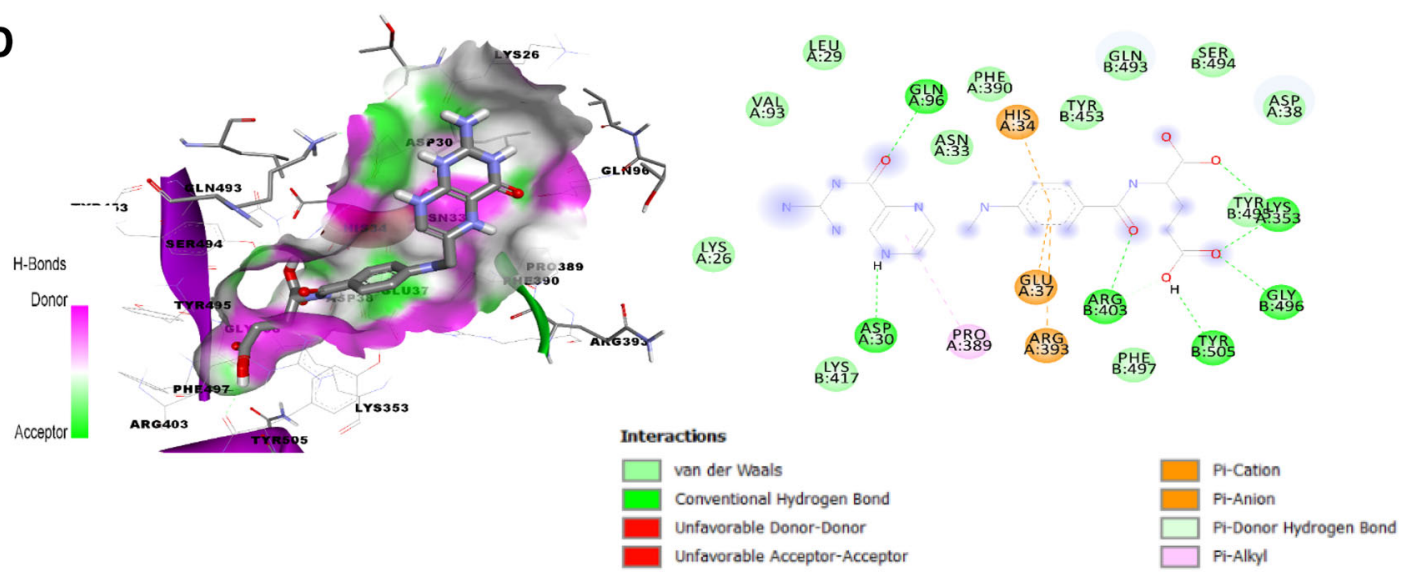


४Fig. 1 Interaction of nutraceuticals with Receptor Binding Domain (RBD) of Spike protein and ACE2 receptor and Papain like protease $\left(\mathrm{PL}^{\mathrm{Pro}}\right)$. a Arbidol interactions with $\mathrm{S}$ protein-ACE-2 complex in the best docked pose. $\mathbf{b}$ Folic acid interactions in the docked complex of $\mathrm{S}$ protein-ACE2. c GRL0617-PL ${ }^{\text {Pro }}$. d 5-Methyltetrahydrofolic acid$\mathrm{PL}^{\text {Pro }}$

following the rules taken maximum two violations of any of the two rules mentioned above (Supplementary Table 2).

\section{Folic acid and it's derivative and Ginkgolide A against $\mathbf{P L}^{\text {pro }}$}

$\mathrm{PL}^{\mathrm{pro}}$, a crucial papain-like protease of the SARS-CoV2, helps in the cleavage of viral polyproteins and making them functional. The inhibition of the functional activity of this protease could inhibit the viral replication inside the host cell. So, the X-ray resolved $(2.7 \AA)$ 3D structure of Apo PL $^{\text {pro }}$ was retrieved from RCSB-PDB database (PDB: $6 \mathrm{~W} 9 \mathrm{C})$. Further, the virtual screening was done with all the 106 nutraceuticals compounds along with GRL0617, an already known hit against PL ${ }^{\text {pro }}$, by generating the grid at the substrate binding site. Grid was generated around main catalytic residues ASP164, VAL165, CYS270, LYS274, VAL303 at catalytic site [38] for docking of compounds. The center of the Grid was $X=-30.19, Y=31.61$, $Z=31.04$ with dimension of 25 Angstrom $^{3}$. After screening, it was found that GRL0617 had the binding score of $-6.2 \mathrm{kcal} / \mathrm{mol}$ and it was making pi-pi interactions with PRO248 and TYR264 and Pi-anion bond with ASP164 in the best binding pose (Fig. 1c). While in the nutraceuticals, 5-methyl-hydrofolic acid had the best binding energy $(-7.1 \mathrm{kcal} / \mathrm{mol})$ better than GRL0617, followed by Ginkgolide-A $(-7.0 \mathrm{kcal} / \mathrm{mol})$, a terpene lactone compound mainly found in Ginkgo biloba and Folic acid $(-6.9 \mathrm{kcal} / \mathrm{mol})$. The 5methylhyrofolic acid had the highest number of hydrogen bond interactions with residues (ASP164, ARG166, ALA246, THR301) at the catalytic site of $\mathrm{PL}^{\text {pro }}$ (Fig. 1d). While, Gingkoglide-A was making the hydrogen bond with GLY163, in the best binding pose. And, Folic acid was making the hydrogen bond with GLY163 and ASP164 (Supplementary Fig. 2C).The ASP164 has been reported to be a main catalytic residue of $\mathrm{PL}^{\text {pro, }}$ which helps in proteolysis of the substrate (Supplementary Fig. 2D). Here we could see that all tree best compounds had multiple polar as well as non-polar interactions at the catalytic site, but 5-methyl-hydrofolic acid and Folic acid were making contact with ASP164. Further in the ADMET analysis Ginkgolide-A was found to better than other top hit nutraceutical compounds as it was not violating any rule of Lipinski and Ghose, while all other molecules were also in the category in drug like molecules (Supplementary Table 2). All these analyses were again indicating the value of the folic acid and its derivative and Ginkgolide-A potential against the inhibition of COVID-19 (Supplementary Table 3).

\section{Water-soluble vitamins against $\mathrm{M}^{\text {pro }}$ of SARS-CoV-2}

Another crucial protease enzyme for SARS-CoV-2 is Main protease or Cysteine like protease, which helps in the cleavage of polyproteins and make them functional. The $\mathrm{X}$-ray resolved structure $(2.16 \AA)$ of the main protease docked with an N3 inhibitor at the substrate-binding site was downloaded from RCSB-PDB database (PDB: 6LU7). The main interacting residues of N3 was taken for creating a grid for the molecular docking of 106 Nutraceutical compounds. So, Grid was generated around PHE140, ASN142, GLY143, HIS164 and Centre of grid was, $X=-19.50, Y=19.24, Z=64.35$; the dimension was $25 * 25 * 25$ Angstrom. Pimozide an already established antipsychotic drug, was found to be an efficient hit with IC50 of $42 \pm 2 \mu \mathrm{mol} / \mathrm{L}$ taken as positive control. After docking based screening, it was found that, Pimozide had the docking score of $-8.0 \mathrm{kcal} / \mathrm{mol}$ and it was making hydrogen bond with ASN33 and HIS34 in the best docking pose (Fig. 2a). While, Magnesium Ascorbate, a buffered (non-acidic) form of Vitamin C (Ascorbic acid), was found to be the top lead compound among 106 nutraceuticals. It had binding energy of $-8.1 \mathrm{kcal} / \mathrm{mol}$, identical to Pimozide and was making the hydrogen bond with LEU141, SER144 and HSI164 (Fig. 2b). Followed by Folic acid and Riboflavin had a similar binding affinity towards $\mathrm{M}^{\text {pro }}$ with the binding energy of $-7.7 \mathrm{kcal} / \mathrm{mol}$. Folic was making polar contact with TYR54, ASP187, ARG188 and THR190 (Supplementary Fig. 3A), while Riboflavin with LEU141, GLY143, CYS145 in their best binding pose at the catalytic site of $\mathrm{M}^{\text {pro }}$ (Supplementary Fig. 3B). All the top hits nutraceuticals along with Pimozide were found to be similar in the drug-likeness property analysis (Supplementary Table 2).Virtual screening of the nutraceutical against $\mathbf{M}^{\text {pro }}$ shown that water-soluble vitamins could be helpful in preventing the functional activity of the enzyme and hence COVID19 infection (Supplementary Table 3).

\section{Folic acid and its derivative and NADH can prevent the endoribonuclease activity}

Once the virus enters inside the host cell, it uses the host translational machinery to produce various Nsps, which helps virus replication. One such Nsp is Nsp15, which is RNA uridylate specific endoribonuclease associated with the viral RNA processing. Inhibition of the functional activity of Nsp15 could help in the inhibition of viral 

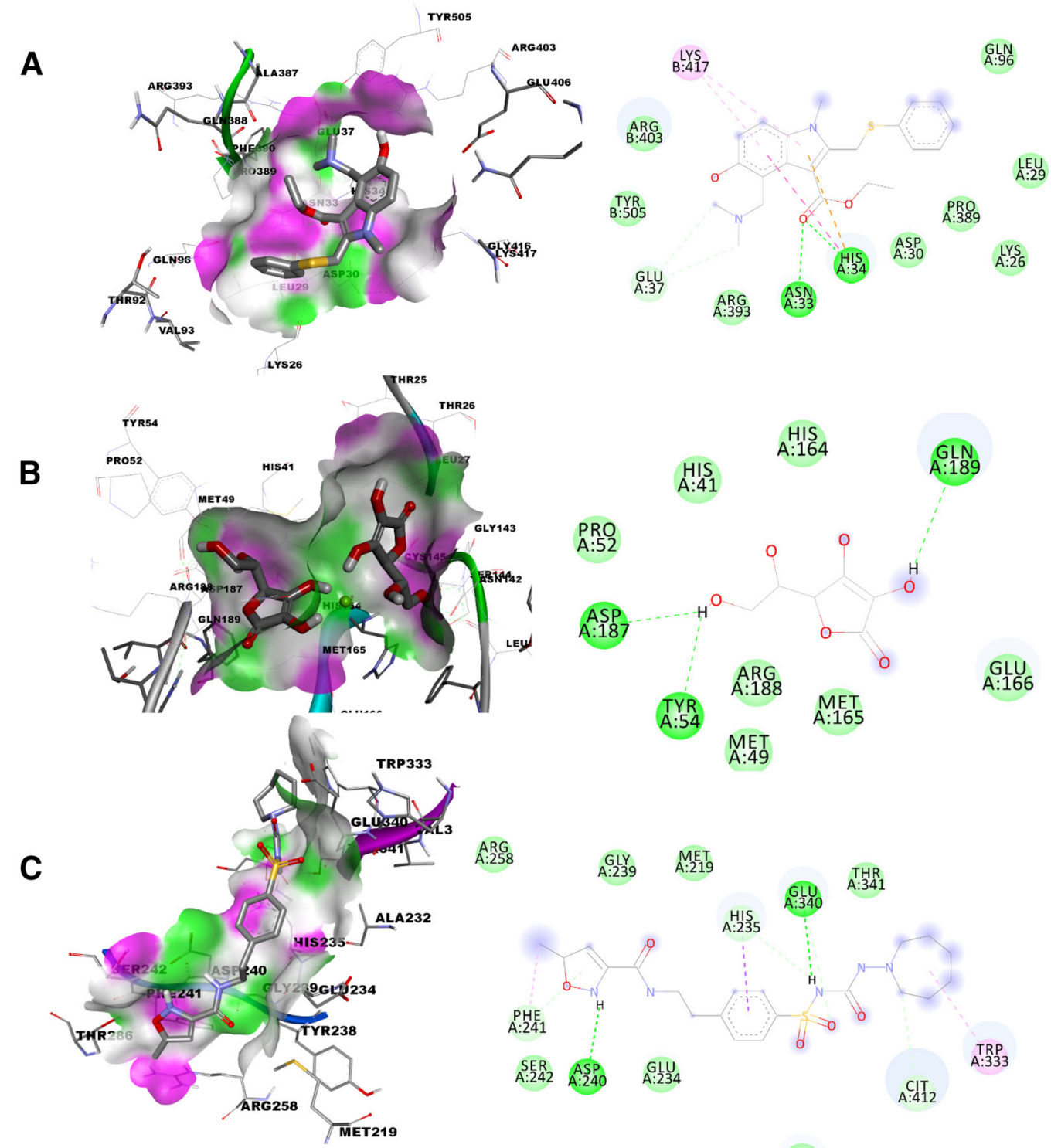

D

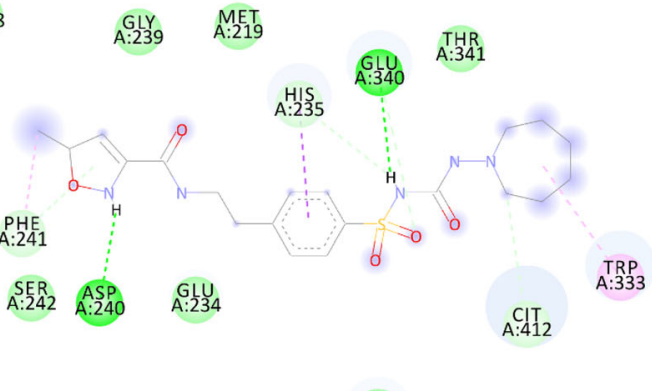

A A: 240

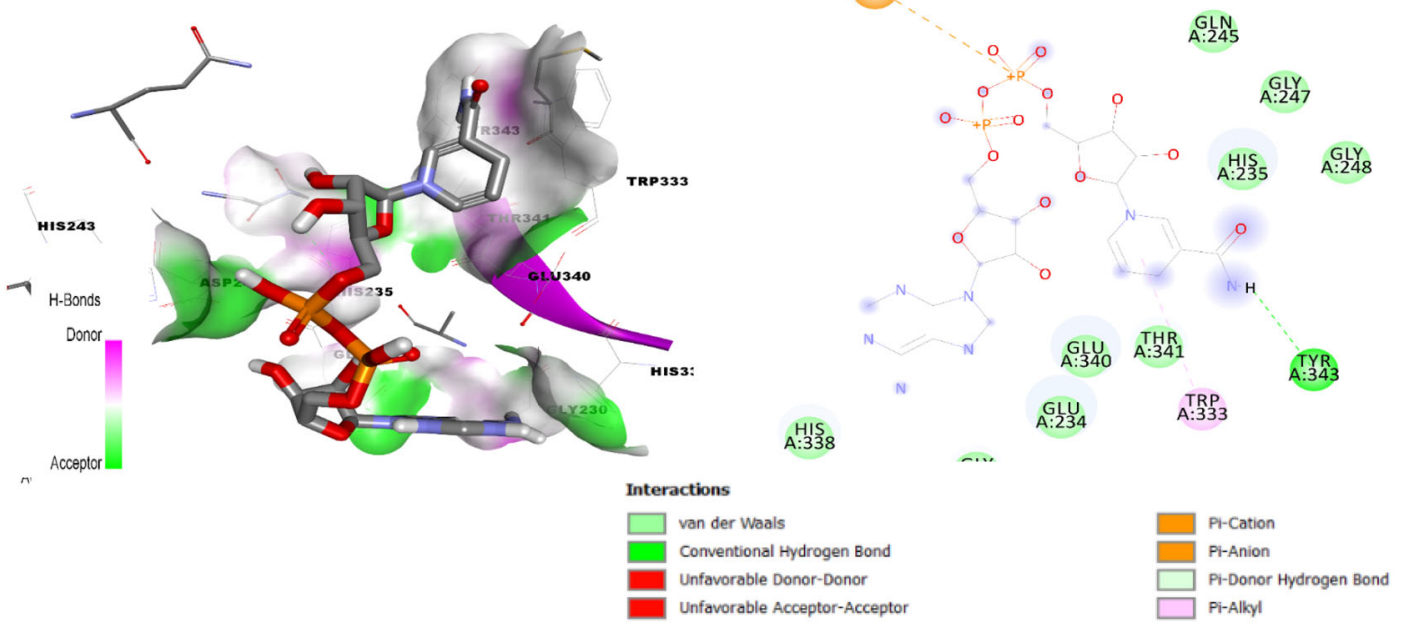

Fig. 2 Interactions of nutraceuticals with SARS-CoV-2 Main protease $\left(\mathrm{M}^{\text {pro }}\right)$ and Nsp15. a Pimozide-M ${ }^{\text {pro }}$. b Magnesium Ascorbate-M ${ }^{\text {pro }}$. c Glisoxepide-Nsp15. d Nicotinamide adenine dinucleotide-Nsp15 
replication inside the body. The 3D structure resolved by X-ray crystallography at the resolution of $1.9 \AA$ was retrieved from RCSB-PDB database (PDB: 6W01). Further, the grid was generated at the substrate-binding site, grid was generated around LEU246, GLY248, LEU249, TYR343 [36]. The center of grid was $X=-62.80$, $Y=67.89, Z=34.93$ and the dimension was $25 * 25 * 25$ Angstrom. The result of the screening shown that, Glisoxepide an already know hit compound against NSP15 had the binding score of $-7.7 \mathrm{kcal} / \mathrm{mol}$ and in the best binding pose it was making hydrogen bon with ASP240 and GLU340 (Fig. 2c). While in case of screened nutraceutical,NADH, Folic acid, and 5-methyl-hydrofolic acid with the binding energy of $-7.2,7.1$ and $7.1 \mathrm{kcal} /-$ mol, respectively, equivalent to Glisoxepide, were the top lead compounds against Nsp15. At the best binding pose, NADH was making one hydrogen bond with TYR343 (Fig. 2d). Folic acid had 3 three hydrogen bond interactions with ASP240, HIS243, and TYR343 (Supplementary Fig. 3C). 5-methyl-hydrofolic acid was also making 3 hydrogen bonds with ASP240, GLU340 and TYR343 (Supplementary Fig. 3D). While the drug likeness properties analysis shown that NADH was violating three rules of Lipinski and four rules of the Ghose et al., hence was found a weak drug candidate, while other compounds were showing drug like properties (Supplementary Table 2). These binding energy and number of interacting residues together were indicating that folic acid and its derivatives had a good binding affinity towards Nsp15, which could be lead compounds in the prevention of COVID-19. (Supplementary Table 4).

\section{Discussion}

The computational methods are considered as the quick and reliable way to predict the action of the drugs against various targets. The recent pandemic situation around the globe has given a specific requirement for innovating new drugs and repurposing the existing drugs against COVID19. The recent availability of the crystal structures of various structural and non-structural proteins of SARSCoV-2 has given the opportunity for structure-based screening of existing compounds against them. Various studies on molecular docking-based screening of existing antiviral, antibacterial and other FDA approved drugs and phytochemicals has already been done and further experimental analysis is still going on. Although the less attention has been given to nutraceutical compounds, which could serve better in the pandemic situation mainly in the poor and developing countries. Taking these points into the considerations we tried investigating the binding affinities of the nutraceutical molecules against various SARS-CoV-
2 and found good binding affinity vitamins and their derivatives. The screened nutraceutical molecules were then also compared with the already known hit against target proteins in terms of docking score, molecular interactions and drug likeness properties. In the case of SpikeACE2 protein interaction inhibition, the top hit nutraceutical was found to be folic acid, whose binding affinity and it interaction was better than already known clinical trial drug Arbidol (Umifenovir). Similarly for $\mathrm{PL}^{\text {pro }}$ too, the already know hit GRL0617 (IC50 $=2.2 \pm 0.3 \mu \mathrm{mol} / \mathrm{L}$ ) had the binding energy of $-6.2 \mathrm{kcal} / \mathrm{mol}$ while the best docking based screened nutraceutical, 5-methyl-hydrofolic acid had the docking score of $7.1 \mathrm{kcal} / \mathrm{mol}$ with better hydrogen bonding. Further in case of $\mathrm{M}^{\text {pro }}$, the docking energy of top screened nutraceutical compound, Magnesium Ascorbate was better than already known hit compound Pimozide (IC50 $=42 \pm 2 \mu \mathrm{mol} / \mathrm{L}$ ). Furthermore in case of NSP15 too, the best screened molecule NADH and folic acid derivetives had similar docking energy as the already known hit drug Glisoxepide (IC50 $=9 \mu \mathrm{mol} / \mathrm{L}$ ). Finally, all the top hit nutraceutical compounds had the identical drug likeness properties as the positive control, indicating the safe use of the hit compounds as drugs. However, in this study, the major limitation is validation of the stability of the docked screened molecules with molecular dynamics simulations and experimental assays due to inaccessibility of the resources in this lockdown period. But we have explored the multitarget ability of these nutraceutical compounds against four main therapeutic targets of SARS-CoV2 through structure based molecular docking which could serve as starting point for further validations, complete list of screened nutraceuticals based on docking score has been provided in Supplementary material $1-4$. The screening results have shown that folic acid alone or with combination with its derivates tetrahydrofolic acid and 5-methyl-tetrahydrofolic acid could be potential molecules against the COVID-19 infection. This computational study strongly suggests a need to investigate folic acid and its derivatives through in vitro and in vivo assays against SARS-CoV-2. In this current pandemic situation and unavailability of the drugs, this cheap and safe alternative can provide a significant value against the combat of COVID-19.

\section{References}

1. Alsaadi EAJ, Jones IM. Membrane binding proteins of coronaviruses. Future Virol. 2019;14(4):275-86. https://doi.org/10. 2217/fvl-2018-0144.

2. Annunziata G, et al. Resveratrol as a novel anti-herpes simplex virus nutraceutical agent: an overview. Viruses. 2018. https://doi. org/10.3390/v10090473. 
3. Banerjee N, Mukhopadhyay S. Viral glycoproteins: biological role and application in diagnosis. Virusdisease. 2016;27(1):1-11. https://doi.org/10.1007/s13337-015-0293-5.

4. Bertram S, et al. Cleavage and activation of the severe acute respiratory syndrome coronavirus spike protein by human airway trypsin-like protease. J Virol. 2011;85(24):13363-72. https://doi. org/10.1128/JVI.05300-11.

5. Bhardwaj $\mathrm{K}$, et al. The coronavirus endoribonuclease Nsp15 interacts with retinoblastoma tumor suppressor protein. J Virol. 2012;86(8):4294-304. https://doi.org/10.1128/JVI.07012-11.

6. BIOVIA, D.S., Discovery studio modeling environment. 2020.

7. Chandra A, et al. Identification of potential inhibitors of SARSCOV-2 endoribonuclease (EndoU) from FDA approved drugs: a drug repurposing approach to find therapeutics for COVID-19. J Biomol Struct Dyn. 2020. https://doi.org/10.1080/07391102. 2020.1775127.

8. Chase AJ, Semler BL. Viral subversion of host functions for picornavirus translation and RNA replication. Future Virol. 2012;7(2):179-91. https://doi.org/10.2217/fvl.12.2.

9. Chen X, Yu B. First two months of the 2019 Coronavirus Disease (COVID-19) epidemic in China: real-time surveillance and evaluation with a second derivative model. Glob Health Res Policy. 2020;5:7. https://doi.org/10.1186/s41256-020-00137-4.

10. Coronaviridae Study Group of the International Committee on Taxonomy of Viruses. The species severe acute respiratory syndrome-related coronavirus: classifying 2019-nCoV and naming it SARS-CoV-2. Nat Microbiol. 2020;5(4):536-44. https://doi.org/ 10.1038/s41564-020-0695-z.

11. Daina A, Michielin O, Zoete V. SwissADME: a free web tool to evaluate pharmacokinetics, drug-likeness and medicinal chemistry friendliness of small molecules. Sci Rep. 2017;7:42717. https://doi.org/10.1038/srep42717.

12. Dallakyan S, Olson AJ. Small-molecule library screening by docking with PyRx. Methods Mol Biol. 2015;1263:243-50. https://doi.org/10.1007/978-1-4939-2269-7_19.

13. Dong L, Hu S, Gao J. Discovering drugs to treat coronavirus disease 2019 (COVID-19). Drug Discov Ther. 2020;14(1):58-60. https://doi.org/10.5582/ddt.2020.01012.

14. Du L, et al. The spike protein of SARS-CoV-a target for vaccine and therapeutic development. Nat Rev Microbiol. 2009;7(3):226-36. https://doi.org/10.1038/nrmicro2090.

15. Fehr AR, Perlman S. Coronaviruses: an overview of their replication and pathogenesis. Methods Mol Biol. 2015;1282:1-23. https://doi.org/10.1007/978-1-4939-2438-7_1.

16. Feng G, et al. COVID-19 and liver dysfunction: current insights and emergent therapeutic strategies. J Clin Transl Hepatol. 2020;8(1):18-24. https://doi.org/10.14218/jcth.2020.00018.

17. Gao X, et al. Crystal structure of SARS-CoV-2 papain-like protease. Acta Pharm Sin B. 2020. https://doi.org/10.1016/j.apsb. 2020.08.014.

18. Ghose AK, Viswanadhan VN, Wendoloski JJ. A knowledgebased approach in designing combinatorial or medicinal chemistry libraries for drug discovery. 1. A qualitative and quantitative characterization of known drug databases. J Comb Chem. 1999;1(1):55-68. https://doi.org/10.1021/cc9800071.

19. Gorton HC, Jarvis K. The effectiveness of vitamin C in preventing and relieving the symptoms of virus-induced respiratory infections. J Manip Physiol Ther. 1999;22(8):530-3. https://doi. org/10.1016/s0161-4754(99)70005-9.

20. Helal NA, et al. Nutraceuticals' novel formulations: the good, the bad, the unknown and patents involved. Recent Pat Drug Deliv Formul. 2019;13(2):105-56. https://doi.org/10.2174/ 1872211313666190503112040 .

21. Hemila H. Vitamin C and infections. Nutrients. 2017. https://doi. org/10.3390/nu9040339.
22. Hoffmann M, et al. SARS-CoV-2 cell entry depends on ACE2 and TMPRSS2 and is blocked by a clinically proven protease inhibitor. Cell. 2020. https://doi.org/10.1016/j.cell.2020.02.052.

23. Jin Z, et al. Structure of M(pro) from COVID-19 virus and discovery of its inhibitors. Nature. 2020. https://doi.org/10.1038/ s41586-020-2223-y.

24. Kim Y, et al. Vitamin $\mathrm{C}$ is an essential factor on the anti-viral immune responses through the production of interferon-alpha/beta at the initial stage of influenza a virus (H3N2) infection. Immune Netw. 2013;13(2):70-4. https://doi.org/10.4110/in.2013. 13.2.70.

25. Kim Y, et al. Crystal structure of Nsp15 endoribonuclease NendoU from SARS-CoV-2. Protein Sci. 2020;29(7):1596-605. https://doi.org/10.1002/pro.3873.

26. Kolifarhood G, et al. Epidemiological and clinical aspects of COVID-19; a narrative review. Arch Acad Emerg Med. 2020;8(1):e41.

27. Le Page M, Hamzelou J. What you need to know. New Sci. 2020;245(3272):8-9. https://doi.org/10.1016/S02624079(20)30475-9.

28. Li H, et al. Coronavirus disease 2019 (COVID-19): current status and future perspectives. Int J Antimicrob Agents. 2020. https:// doi.org/10.1016/j.ijantimicag.2020.105951.

29. Lim YX, et al. Human coronaviruses: a review of virus-host interactions. Diseases. 2016. https://doi.org/10.3390/ diseases4030026.

30. Lipinski CA, et al. Experimental and computational approaches to estimate solubility and permeability in drug discovery and development settings. Adv Drug Deliv Rev. 2001;46(1-3):3-26. https://doi.org/10.1016/s0169-409x(00)00129-0.

31. Lu R, et al. Genomic characterisation and epidemiology of 2019 novel coronavirus: implications for virus origins and receptor binding. Lancet. 2020;395(10224):565-74. https://doi.org/10. 1016/S0140-6736(20)30251-8.

32. Morrey JD, et al. Effects of folic acid malnutrition on rotaviral infection in mice. Proc Soc Exp Biol Med. 1984;176(1):77-83. https://doi.org/10.3181/00379727-176-41845.

33. Nakagawa K, Lokugamage KG, Makino S. Viral and cellular mRNA translation in coronavirus-infected cells. Adv Virus Res. 2016;96:165-92. https://doi.org/10.1016/bs.aivir.2016.08.001.

34. Nasri $\mathrm{H}$, et al. New concepts in nutraceuticals as alternative for pharmaceuticals. Int J Prev Med. 2014;5(12):1487-99.

35. Nichols WG, Peck Campbell AJ, Boeckh M. Respiratory viruses other than influenza virus: impact and therapeutic advances. Clin Microbiol Rev. 2008;21(2):274-90. https://doi.org/10.1128/cmr. 00045-07.

36. Ortiz-Alcantara J, Bhardwaj K, Palaninathan S, Frieman M, Baric RS, Kao CC. Small molecule inhibitors of the SARS-CoV Nsp15 endoribonuclease. Virus Adapt Treat Virus Adapt Treat. 2010;2:125-33.

37. Ricagno $S$, et al. Crystal structure and mechanistic determinants of SARS coronavirus nonstructural protein 15 define an endoribonuclease family. Proc Natl Acad Sci USA. 2006;103(32):11892-7. https://doi.org/10.1073/pnas. 0601708103.

38. Rimanshee, A., et al., Potential inhibitors against papain-like protease of novel coronavirus (SARS-CoV-2) from FDA approved drugs. 2020.

39. Singhal T. A review of Coronavirus Disease-2019 (COVID-19). Indian J Pediatr. 2020;87(4):281-6. https://doi.org/10.1007/ s12098-020-03263-6.

40. te Velthuis AJ, et al. The RNA polymerase activity of SARScoronavirus nsp12 is primer dependent. Nucl Acids Res. 2010;38(1):203-14. https://doi.org/10.1093/nar/gkp904.

41. Trott $\mathrm{O}$, Olson AJ. AutoDock Vina: improving the speed and accuracy of docking with a new scoring function, efficient 
optimization, and multithreading. J Comput Chem. 2010;31(2):455-61. https://doi.org/10.1002/jcc.21334.

42. Tyrrell DAJ, Myint SH. Coronaviruses. In: Baron S, editor. Medical microbiology. Galveston, TX: University of Texas Medical Branch at Galveston; 1996.

43. van Woensel JB, van Aalderen WM, Kimpen JL. Viral lower respiratory tract infection in infants and young children. BMJ. 2003;327(7405):36-40. https://doi.org/10.1136/bmj.327.7405.36.

44. Vatansever, E.C., et al., Targeting the SARS-CoV-2 main protease to repurpose drugs for COVID-19. bioRxiv. 2020.

45. Wang Q, et al. Structural and functional basis of SARS-CoV-2 entry by using human ACE2. Cell. 2020;181(4):894-904. https:// doi.org/10.1016/j.cell.2020.03.045.

46. Watanabe Y, et al. Exploitation of glycosylation in enveloped virus pathobiology. Biochim Biophys Acta Gen Subj. 2019;1863(10):1480-97. https://doi.org/10.1016/j.bbagen.2019. 05.012.

47. Xu P, et al. Arbidol/IFN-alpha2b therapy for patients with corona virus disease 2019: a retrospective multicenter cohort study. Microbes Infect. 2020;22(4-5):200-5. https://doi.org/10.1016/j. micinf.2020.05.012.
48. Yamshchikov AV, et al. Vitamin D for treatment and prevention of infectious diseases: a systematic review of randomized controlled trials. Endocr Pract. 2009;15(5):438-49. https://doi.org/ 10.4158/EP09101.ORR.

49. Yang Y, et al. The deadly coronaviruses: the 2003 SARS pandemic and the 2020 novel coronavirus epidemic in China. J Autoimmun. 2020;109:102434. https://doi.org/10.1016/j.jaut.2020. 102434.

50. Yi Y, et al. COVID-19: what has been learned and to be learned about the novel coronavirus disease. Int $\mathrm{J}$ Biol Sci. 2020;16(10):1753-66. https://doi.org/10.7150/ijbs.45134.

51. Zahra, S., et al., The role of folic acid in the management of respiratory disease caused by COVID-19. 2020.

52. Zhou G, Zhao Q. Perspectives on therapeutic neutralizing antibodies against the Novel Coronavirus SARS-CoV-2. Int J Biol Sci. 2020;16(10):1718-23. https://doi.org/10.7150/ijbs.45123.

Publisher's Note Springer Nature remains neutral with regard to jurisdictional claims in published maps and institutional affiliations. 